\title{
Quand le BMS a une longueur d'avance
}

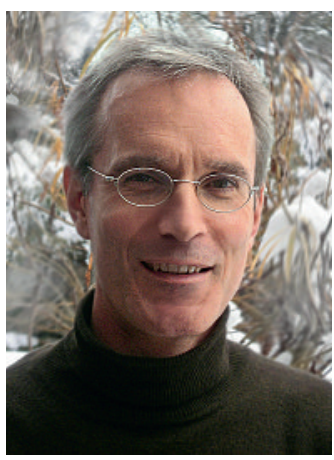

Bruno Kesseli
Lorsque des journalistes discutent de la pertinence journalistique d'un thème, par exemple dans le cadre d'une séance de rédaction, le «lecteur» (homme ou femme) est un hôte très apprécié en tant qu'élément de l'instrumentaire d'argumentation. «Cela n'intéresse pas le lecteur», «le lecteur ne va pas suivre» ou «c'est justement ce que le lecteur veut savoir» sont des formulations que l'on pourrait presque qualifier de «classiques».

Bien entendu, tout cela n'a pas grand-chose à voir avec «le lecteur» justement. Il s'agit seulement de défendre sa propre position (question hérétique: s'agit-il véritablement toujours du bien des patients lorsque nous, les médecins, le mettons au premier plan?). En dépit de méthodes toujours plus raffinées d'étude du public - on parle déjà de «lecteurs transparents», malgré des forums mis en place par les médias et très utilisés ainsi que des possibilités de contact par courrier électronique très faciles avec les rédactions, la distance entre les médias et leur public reste évidente. Et de plus, dans notre société pluraliste, le «lecteur» n'existe pas en tant que tel. sommes assez réalistes pour savoir que l'on ne peut jamais plaire à tout le monde. Mais l'objectif de la rédaction doit être et rester à l'avenir que, chaque semaine, chaque médecin du pays se sente concerné par l'un ou l'autre article du BMS. Même si le BMS possède, selon diverses enquêtes indépendantes, une excellente portée, le potentiel dans ce domaine n'est certainement pas encore épuisé.

Il existe encore un autre aspect fondamental par lequel le BMS se distingue de la presse grand public: nos lecteurs sont aussi nos auteurs. Même si nous nous efforçons de développer un profil original grâce aux articles réguliers des membres de la rédaction et de journalistes indépendants, outre la fonction d'organe officiel de la FMH, l'une des missions principales du BMS est de permettre aux médecins de s'exprimer, dans toute leur diversité.

Cette partie de notre travail ne s'est pas allégée l'année dernière, au contraire: le vent contraire auquel le secteur de la presse écrite est exposé d'un point de vue économique a également soufflé sur le BMS. Il a fallu réduire le nombre de pages rédactionnelles dis-

\section{L'objectif de la rédaction doit être que, chaque semaine, chaque méde- cin du pays se sente concerné par l'un ou l'autre article du BMS}

Ce n'est pas que le Bulletin des Médecins Suisses (BMS) soit différent des autres. Mais il possède tout de même certains avantages en matière de proximité du lecteur par rapport aux médias grand public. Il s'agit d'une part du fait que les rédacteurs du BMS connaissent directement le milieu professionnel de leur lectorat ou en font part dans la mesure où ils sont ou ont été eux-mêmes actifs dans le système de santé, le plus souvent en tant que médecins. C'est peut-être l'un des motifs pour lesquels les contacts avec les lecteurs (qui sont aussi des collègues) sont plus fréquents pour le BMS que pour la presse grand public.

Cette année pour la première fois, le BMS a tenu des tables rondes. Ce fut une nouvelle occasion très bienvenue de discuter avec des collègues, dans un contexte agréable, et d'aborder également des questions journalistiques. Il a été aussi réjouissant que motivant de pouvoir constater que les efforts de la rédaction du BMS, afin de concevoir un produit acceptable avec très peu de moyens, sont perçus comme tels et appréciés.

Naturellement, il faut admettre que les réactions dans le cadre de tels contacts sont biaisées. Nous ponibles par numéro pour des raisons de coût. Concrètement, pour les auteurs potentiels, cela signifie que les articles seront plus courts (ce qui souvent n'est pas un inconvénient pour les lecteurs), les temps d'attente plus longs et le taux de refus légèrement plus important.

Bien que, dans certains cas, le débat puisse être difficile, la compréhension des décisions rédactionnelles est en général bonne et la collaboration avec les collègues auteurs constructive et enrichissante. On comprend généralement qu'avec largement plus de mille articles proposés chaque année, tous ne peuvent être acceptés et qu'il existe bien sûr un délai de publication même si l'on considère naturellement toujours que son propre texte est important et doit être placé comme prioritaire.

Dans ce contexte, je me réjouis de poursuivre la collaboration au sein de la rédaction et avec vous, lecteurs et auteurs potentiels! Je saisis l'occasion du dernier numéro de l'année pour vous souhaiter à tous de joyeuses fêtes et une excellente année nouvelle.

Bruno Kesseli, rédacteur en chef 